\title{
A Load Balancing Uneven Clustering approach for wireless sensor networks
}

\author{
Y. L. Jia ${ }^{1, a *}$, C. Y. Zhang ${ }^{1, b}$ \\ ${ }^{1}$ College of Automation Engineering, Nanjing University of Aeronautics and Astronautics, Nanjing \\ china \\ aflybearnuaa@163.com, ${ }^{\mathrm{b}}$ zhangchiyu01@126.com
}

\begin{abstract}
Keywords: Wireless sensor networks; Distributed approach; Clustering; Network lifetime; Energy efficiency

Abstract. Energy consumption is critical to wireless sensor networks (WSNs), and cluster based protocols are energy efficient. Cluster heads (CHs) selection and clustering affect the network lifetime greatly. To prolong the network lifetime, a load balancing unequal clustering approach (LBUC) is proposed. LBUC forms unequal clusters to balance the energy consumption among the $\mathrm{CHs}$ and the data is transmitted to base station (BS) by multi-hop. The CHs are selected according to the residual energy and the distance to neighbor nodes. LBUC restricts the CHs count and the max nodes count of a cluster. The experimental results indicate that LBUC forms appropriate clusters and prolongs the network lifetime.
\end{abstract}

\section{Introduction}

WSNs, made by a large count of nodes in the monitoring area, are formed by the way of wireless communication and widely used in the field of environmental monitoring. In general, node energy is limited, therefore how to design low power consumption and high reliability algorithm for WSNs is important [1].

Cluster based routing protocol can effectively save energy and prolong the network lifetime [2]. In cluster based routing protocol, some nodes act as the $\mathrm{CHs}$. Other nodes sense and translate data to the CHs. The CHs collect and fuse data, then translate data to the BS [3].

This paper proposes a novel distributed approach named LBUC for WSNs. The rest of the paper is organized as follows. Sect. 2 synopsizes the related work. Sect. 3 gives Network models. Sect. 4 presents LBUC in detail. Sect. 5 discusses the simulation results. Sect. 6 gives the conclusions.

\section{Related Work}

LEACH is a classical cluster based protocol, and the whole network is divided into several clusters. In this algorithm, the time period is divided into several rounds, each round is divided into initial stage and stable stage. The $\mathrm{CHs}$ are selected and the clusters are formed in initial stage and the nodes gather and translate the data in stable stage. The selection of CHs is random, and LEACH has nothing to do with the residual energy or the locations of the nodes so the lifetime is short [4].

Due to the randomness present in LEACH, count of $\mathrm{CHs}$ generated varies highly from the optimal count. Ref. [5] presents an approach named LEACH-MAC which attempts to control the randomness present in LEACH. When selecting CHs, the network keeps on selecting until the count of selected $\mathrm{CHs}$ is equal to the optimal count. This approach makes the $\mathrm{CHs}$ count stable and prolongs the lifetime.

EEUC uses multi-hop routing based on uneven clustering, through non uniform radius of competition to ensure the $\mathrm{CHs}$ that are close to the BS have fewer nodes in their cluster. On this way, EEUC balances the energy consumption among the CHs [6].

To balance the load between CHs and save the energy consumption of the inter-cluster routing, Ref. [7] proposes an energy-efficient routing algorithm based on uneven clustering. In CHs election, the approach take into consideration the vote based measure and the transmission power of sensor nodes to sectionalize these nodes into different unequal clusters. 
DUCF is an uneven cluster approach. The approach selects $\mathrm{CHs}$ according to two factors: the residual energy and the count of neighbor nodes [8]. DUCF ensures load balancing among the clusters by varying the cluster size of its $\mathrm{CH}$. Here, cluster size is the maximum count of nodes to be its cluster members for a particular $\mathrm{CH}$ and if the node count in a cluster achieves the cluster size, the $\mathrm{CH}$ no longer accept new nodes to join its cluster. DUCF assigns cluster size based on the count of neighbor nodes and the distance to BS. DUCF balance the energy consumption of CHs and prolong the network lifetime.

These algorithms prolong the network lifetime, but also have their own disadvantages. LEACH-MAC makes the CHs count stable, but the node counts of different cluster are varied, so the energy consumptions of CHs are not balanced. DUCF balances load among the clusters but clustering process is complex. Some nodes may fail several times to join a cluster and a node may join a distant $\mathrm{CH}$. In the worst case, a node is unable to join a cluster and must select itself as a $\mathrm{CH}$, so the $\mathrm{CHs}$ count is varied.

\section{Network and Radio Model}

The network has the following properties:

a) The network covers $M \times M \mathrm{~m}^{2}$ region and $N$ nodes evenly distribute in the region.

b) The BS is located in the center of the region and all nodes can communicate with the BS directly.

c) All nodes are stationary and send data to the CHs by single-hop, and the CHs send data to the BS by multi-hop.

d) A node aware itself residual energy but unaware position. A nodes have its own ID, and can fuse, transmit and receive data.

Energy consumed for transmitting $l$ bit data at distance $d$ is calculated as Eq. (1) .

$$
\begin{aligned}
& E_{T x}(l, d)=\left\{\begin{array}{l}
l \times E_{\text {elec }}+l \times \varepsilon_{f s} d^{2}, d<d_{\text {corssover }} \\
l \times E_{\text {elec }}+l \times \varepsilon_{m p} d^{4}, d \geq d_{\text {corssover }}
\end{array}\right. \\
& E_{R x}(l)=l \times E_{\text {elec }}
\end{aligned}
$$

Here, $E_{\text {elec }}$ is the energy consumption per bit to run the transmitter or the receiver circuit, $\varepsilon_{f s}$ is f(b) free space channel model and $\varepsilon_{m p}$ is for multi-path fading channel model, $d_{\text {corssover }}$ is a constant [9].

A receiver can estimate the distance to the sender by comparing the strength of the receiving signal and the signal strength of the sending signal. Nodes can change the transmission power depending on the distance to the receiver.

\section{Proposed Approach}

\section{CHs Selection}

LBUC is a clustering approach and all nodes are partitioned into some clusters and each cluster has a $\mathrm{CH}$. If the $\mathrm{CHs}$ count is different, the network energy consumption is also different. If the count of $\mathrm{CHs}$ is too many, the effect of data fusion is poor, each $\mathrm{CH}$ will send data to the $\mathrm{BS}$ and the energy consumption will increase. If the count of $\mathrm{CHs}$ is too few, the distance between a node to the $\mathrm{CH}$ would be very far, the energy consumption of inter-cluster communication will increase. For an unequal cluster and multi-hop networks, the optimal count of $\mathrm{CHs}$ is $k$ and the calculation formula of $k$ is presented in Ref. [10,11].

In the cluster network, a $\mathrm{CH}$ receives the data from the nodes and sends the data to the $\mathrm{BS}$, the energy consumption of a $\mathrm{CH}$ is relatively large. In order to balance the energy consumption among nodes, the CHs can only be selected in the nodes with large residual energy.

In the multi-hop network, CHs need to transmit the data sent from other CHs. Usually the closer to the $\mathrm{BS}$, the greater amount of data need to transmit by a $\mathrm{CH}$, the higher the energy consumption. In order to balance the energy consumption of the $\mathrm{CHs}$, we use uneven clustering approach. A $\mathrm{CH}$, which is close to the BS, should have fewer nodes in its cluster to reduce the energy consumption of the inter-cluster communication. Because the nodes are evenly distributed, the area of a cluster that is 
close to the BS should be small, so the nodes in the cluster are few. The radius of a cluster is calculated as Eq. (2).

$$
R_{j}=\left(1-g \times \frac{d_{\max }-d_{l}}{d_{\text {max }}-d_{\text {min }}}\right) R_{\max }
$$

Here $R_{j}$ is the radius of $\mathrm{CH} j, R_{\max }$ is the maximum radius that is predefined, $d_{j}$ is the distance between $j$ and the BS, $d_{\max }$ and $d_{\min }$ are the maximum and the minimum value of $d_{j}, g$ is a constant coefficient and $g \in(0,1)$. Usually $g$ is 0.5 .

If the distance between a node to $j$ is less than $R_{j}$, the node is a neighbor node of $j$. We assume $d_{\text {avg }}$ is the average distance from the neighbor nodes to $j$. If $d_{\text {avg }}$ is small, the energy consumption of inter-cluster communication is small. When CHs selection starting, each node calculates $t_{i}$ and start waiting.

$$
t_{i}=\operatorname{MAX}\left(0, t_{0}-t_{0} \frac{E_{\text {res }}}{E_{\text {avg }}}\right)+\operatorname{MIN}\left(t_{0}, t_{0} \frac{d_{\text {avg }}}{R_{j}}\right)
$$

Here $t_{0}$ is a predefined interval, $E_{\text {avg }}$ is the average residual energy, $E_{\text {res }}$ is the residual energy(bf) node $i$. When the waiting is end, a node becomes a $\mathrm{CH}$ for the current round if the $\mathrm{CHs}$ selection is not over. Then the node broadcasts a CLUSTER-FORM message with its ID to other nodes. If the BS has receives $k$ CLUSTER-FORM messages or $2 t_{0}$ is over, the BS broadcasts a SELECT-END message to end the CHs selection.

\section{Nodes Clustering}

We assume $n$ as the optimal count of the nodes in a cluster.

$$
n=N \frac{\pi R_{j}^{2}}{M^{2}}
$$

If the count of nodes in a cluster is less than $n$, new nodes will be allowed to join the cluster, otherwise, the $\mathrm{CH}$ will refuse new nodes to join the cluster. When clustering, after $\mathrm{CH}$ selected, a node set a waiting time $t_{q}$ for each $\mathrm{CH}$.

$$
t_{q}=\frac{l}{R_{j}} t_{1}
$$

Here $l$ is the distance between the node and the $\mathrm{CH}, t_{1}$ is a predefined interval. After $\mathrm{CHs}$ selected, all nodes start to join clusters at the same time. A node set a waiting time for each $\mathrm{CH}$. A $\mathrm{CH}$ accept nodes to join its cluster until the count of nodes in its cluster is $n$, then, it send a MEMBER-FULL message with its ID to indicate no space for the new member node. Before join a cluster, a node wait for $t_{q}$. When waiting, if the node receive the MEMBER-FULL message from the $\mathrm{CH}$, the node no longer choose the $\mathrm{CH}$ and try to join another cluster. If the node doesn't receive the MEMBER-FULL message before $t_{q}$ is over, it sends a JOIN message with its ID to the cluster. In this way, the average distance from the nodes to the $\mathrm{CH}$ is as small as possible. After $t_{1}$, most nodes should join a cluster. If a node doesn't join any cluster yet, it pick a $\mathrm{CH}$ that its cluster is not full at random and sends a JOIN message with its ID to the $\mathrm{CH}$ and the $\mathrm{CH}$ should accept the node.

\section{Step of LBUC}

The Main Steps of LBUC are as follows:

Step 1. At beginning, all nodes including the BS broadcast their residual energy and IDs. A node receives the signals from other nodes, estimate the distances to others and calculates $R_{j}$, and $n$.

Step 2. The BS receives signals, then calculates and broadcasts $E_{a v g}$. Each node receives the message, then calculate the waiting time. The CHs are selected by the way described in Sect. 4.1.

Step 3. Each node chooses a CH to join the cluster by the way described in Sect. 4.2. The CHs receive the JOIN messages, and if it accept the requests, it post a ACCEPT message to the nodes.

Step 4. The nodes gather and transmit the data with their residual energy information to their CHs. The $\mathrm{CHs}$ receive and fuse the data form the nodes and other $\mathrm{CHs}$, then transmit the data with the 
residual energy information to the BS. The BS receives the data. When a round is over, repeat from step 2.

\section{Results and Analysis}

We simulated the LEACH, LEACH-MAC, DUCF and LBUC in the NS2 environment. 200 nodes are randomly placed in a field which size is $200 \mathrm{~m} \times 200 \mathrm{~m}$. The BS is located at the center. The simulation parameters and values are mentioned in Table 1.

Table 1 Simulation parameters and values.

\begin{tabular}{cc}
\hline Parameter & value \\
\hline$\varepsilon_{f s}$ & $10 \mathrm{pJ} / \mathrm{bit} / \mathrm{m}^{2}$ \\
$\varepsilon_{m p}$ & $0.0013 \mathrm{pJ} / \mathrm{bit}^{2} \mathrm{~m}^{4}$ \\
$E_{\text {elec }}$ & $50 \mathrm{~nJ} / \mathrm{bit}$ \\
$d_{\text {corssorer }}$ & $87 \mathrm{~m}$ \\
\hline
\end{tabular}

Fig. 1 shows the whole network energy consumption in a round. The energy consumption of LEACH is the largest and significantly different in different rounds. This is because LEACH takes into account neither the residual energy nor the location. LEACH-MAC selects suitable count of CHs to reduce the energy consumption. DUCF is a multi-hop network and forms clusters with suitable count of nodes, so the energy consumptions are smaller and similar in different rounds. The count of CHs of LBUC is static and the nodes count in a cluster is suitable, so the energy consumption is the smallest and the dissipated energy consumption line is the smoothest in the four approaches.

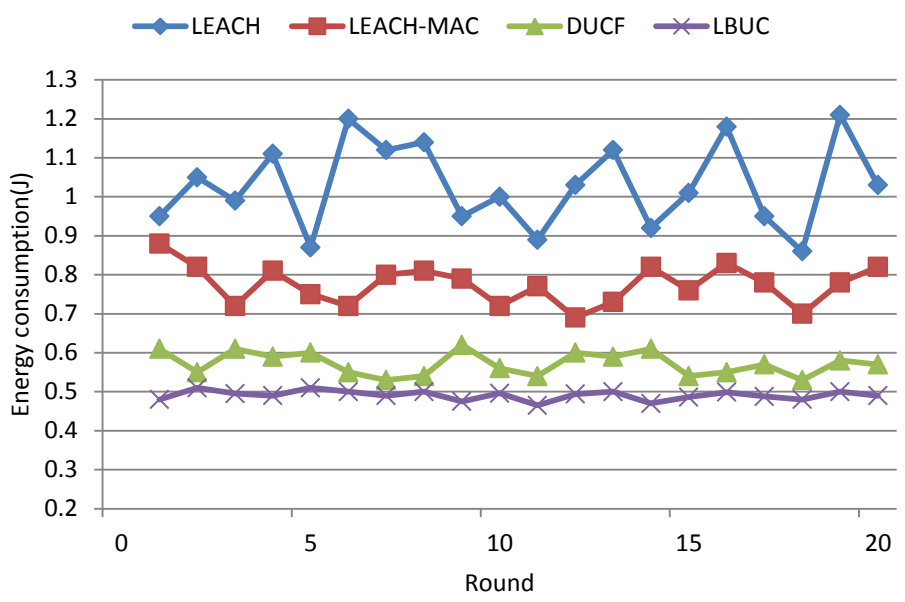

Fig. 1 The whole network energy consumption for a round

Fig. 2 shows the lifetimes of network. The lifetime of LEACH is shortest. LEACH-MAC controls the inherent randomness of the $\mathrm{CH}$ selection and also gives priority to the higher energy node for becoming a $\mathrm{CH}$, so the network lifetime is prolonged. DUCF assigns the maximum count of the nodes for a $\mathrm{CH}$ to balance the energy consumption among $\mathrm{CHs}$ and the lifetime is longer. LBUC also limit the nodes count of a $\mathrm{CH}$, the count of $\mathrm{CHs}$ is stable and the clustering process is simpler compared with DUCF. CHs are selected according to the residual energy and the spacing of the nodes, so the lifetime is the longest.

\section{Conclusion and future works}

The CHs count and the nodes count in a cluster affects the network lifetime greatly. We propose a clustering approach for WSNs. LBUC is a multi-hop approach and clusters the nodes unevenly. LBUC makes the $\mathrm{CHs}$ count stable, balances the energy consumption among different $\mathrm{CHs}$ by restricting the node count, and gives priority to the node that is closer to its neighbor nodes for 
becoming a $\mathrm{CH}$. Simulation results show that LBUC prolongs the network lifetime. As a future work, we will attempt to distribute the CHs evenly in the network.

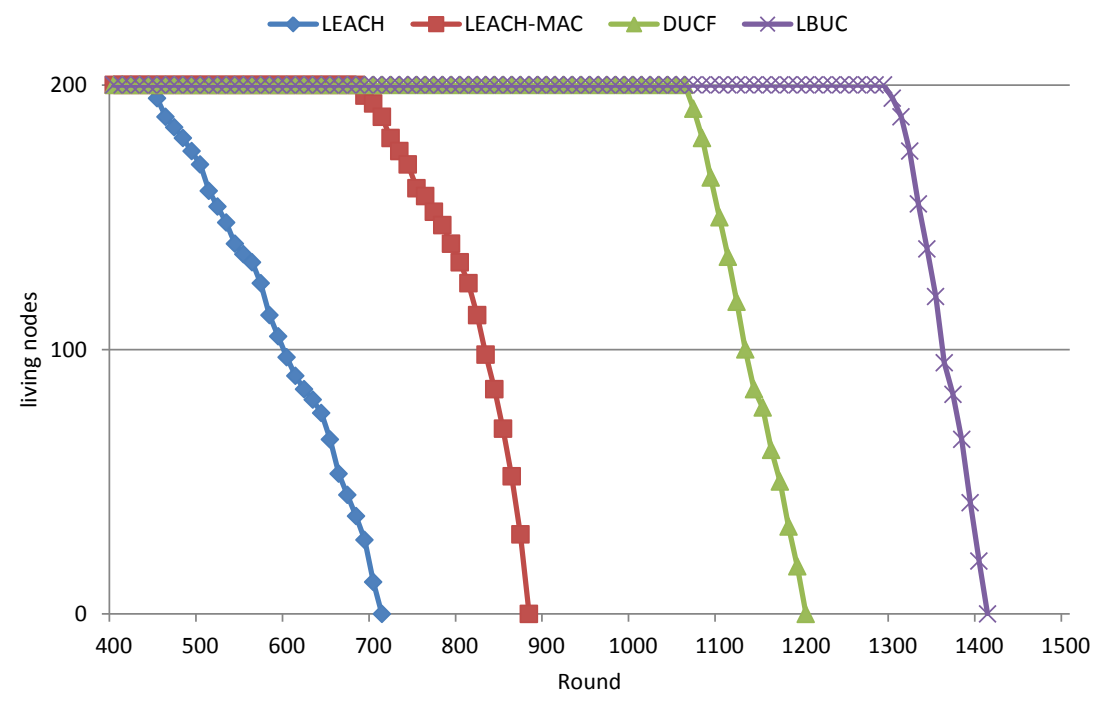

Fig. 2 Network lifetime

\section{References}

1.R. V. Kulkarni, A. Förster, G.K. Venayagamoorthy, Computational Intelligence in Wireless Sensor Networks: A Survey, Communications Surveys \& Tutorials, IEEE, 2011,4(13) :68-96.

2. N. Pantazis, S. Nikolidakis, D.Vergados, Energy efficient routing protocols in wireless sensor networks: A survey, IEEE Communications Surveys \& Tutorials, 2013,15(2), 551-591.

3. A. Abbasi, M. Younis, A survey on clustering algorithms for wireless sensor networks, Computer Communications, 2007,30(14), 2826-2841.

4. M. Afsar, M. Tayarani, Clustering in sensor networks: A literature survey, Journal of Network and Computer Applications,2014,46, 198-226.

5. P. K. Batra, K. Kant, LEACH-MAC: a new cluster head selection algorithm for Wireless Sensor Networks, Wireless Networks, 2016,22(1):49-60.

6. C.F. Li, M. Ye, G.H. Chen, An energy-efficient unequal clustering mechanism for wireless sensor network, in: IEEE International Conference on Mobile Adhoc and Sensor Systems Conference, 2005, pp. 596-640.

7. X. Hui, Z. R. Hua, Y. Jia, Energy-Efficient Routing Algorithm Based on Unequal Clustering and Connected Graph in Wireless Sensor Networks, International Journal of Wireless Information Networks, 2016, 3(11):1-10.

8. B. Baranidharan, B. Santhi, DUCF: Distributed load balancing Unequal Clustering in wirelesssensor networks using Fuzzy approach, Applied Soft Computing, 2016,1(40):495-506.

9. G. Anastasi, M.Conti, M. D. Francesco, Energy conservation in wireless sensor networks: A survey. Ad Hoc Networks, 2009,7(3), 537-568.

10. L. G. Fan, X. duo, A routing protocol based on uneven clustering and path optimization in wireless sensor networks, Computer Engineering and Science, 2015, 37(8):1492-1497.

11. S. J. Shu, G. W. Zhong, Y. C. Long, Fault-Tolerance Clustering Algorithm with Load-Balance Aware in Wireless Sensor Network, Chinese Journal of Computers, 2014, 37(2): 445-456. 